\title{
Instrumentos de Avaliação no Transtorno do Espectro Autista: Uma Revisão Sistemática
}

\author{
Camila Costa e Silva \\ Pontifícia Universidade Católica de Goiás, Goiânia-GO, Brasil \\ Luciana Carla dos Santos Elias \\ Universidade de São Paulo, Ribeirão Preto-SP, Brasil
}

\section{RESUMO}

Este trabalho procurou revisar sistematicamente a literatura científica acerca dos instrumentos validados no Brasil para a caracterização do Transtorno do Espectro Autista (TEA), segundo relato de responsáveis e/ou cuidadores. Realizou-se busca nas bases de dados: Embase, PubMed, Scopus e BVS, obtendo oito artigos publicados entre 2009 e março de 2019. Utilizou-se o protocolo PRISMA e dois juízes verificaram, de forma independente, procedimentos de seleção e extração de artigos. Constatou-se variação nos estudos identificados quanto à amostra, etapas seguidas na validação dos instrumentos, resultados estatísticos obtidos e submissão ao SATEPSI. Embora os instrumentos apresentem evidências de validade, estão disponíveis apenas em trabalhos científicos. Considerando os aspectos psicométricos e as diretrizes do Conselho Federal de Psicologia, não foi identificado um instrumento diagnóstico específico para o TEA disponível ao profissional psicólogo no Brasil, evidenciando uma situação crítica para a prática clínica nessa área.

Palavras-chave: avaliação psicológica; autismo; instrumentos psicológicos.

\section{ABSTRACT - Assessment Tools for Autism Spectrum Disorder: A Systematic Review}

This paper sought to systematically review the scientific literature related to instruments validated in Brazil for the characterization of Autism Spectrum Disorder (ASD), according to reports of parents and/or caregivers. The following databases were searched: Embase, PubMed, Scopus and VHL. Eight articles were published between 2009 and March 2019. The PRISMA protocol was used and two judges independently verified the article selection and inclusion procedures. There were variations in the studies identified regarding the sample, the steps followed in the validation of the instruments, the statistical results obtained and the submission to SATEPSI. Although the instruments present evidence of validity, they are only available in scientific papers. Considering the psychometric aspects and the guidelines of the Federal Council of Psychology, no specific diagnostic instrument for ASD was identified as being available to the professional psychologist in Brazil, demonstrating a critical situation for the clinical practice in this area.

Keywords: Psychological assessment; Autism; Psychological instruments.

RESUMEN - Herramientas de Evaluación del Trastorno del Espectro Autista: una Revisión Sistemática

Este trabajo buscó revisar sistemáticamente la literatura científica con respecto a instrumentos validados en Brasil para la caracterización del Trastorno del Espectro Autista (TEA), según informes de tutores y/o cuidadores. Las búsquedas fueron realizadas en las siguientes bases de datos: Embase, PubMed, Scopus y BVS, resultando en ocho artículos publicados entre 2009 y marzo de 2019. Se utilizó el protocolo PRISMA y dos jueces verificaron de forma independiente los procedimientos de selección y extracción de artículos. Se constató variación en los estudios identificados con respecto a la muestra, las etapas seguidas en la validación de los instrumentos, los resultados estadísticos obtenidos y el envío al SATEPSI. Aunque los instrumentos presentan evidencias de validez, solo están disponibles en artículos científicos. Teniendo en cuenta los aspectos psicométricos y las pautas del Consejo Federal de Psicología, no se identificó ningún instrumento de diagnóstico específico para el TEA disponible para el psicólogo profesional en Brasil, lo que muestra una situación crítica para la práctica clínica en esta área.

Palabras clave: Evaluación psicológica; Autismo; Instrumentos psicológicos.

O Transtorno do Espectro Autista (TEA) é marcado por desordens de origem neurobiológica, sendo os prejuízos referentes aos comportamentos restritivos e repetitivos e déficits na área sociocomunicativa (Júlio-Costa \& Antunes, 2017). A palavra "autismo" foi empregada pela primeira vez em 1911 por Bleuler, um psiquiatra suíço. A caracterização do transtorno é marcada por reformulações no Manual Diagnóstico Estatístico de Transtornos Mentais (DSM). Inicialmente no DSM I o autismo foi considerado sintoma da "Reação Esquizofrênica, tipo infantil”; na sequência no DSM II a categoria passou a ser descrita como "Esquizofrenia, 
do tipo infantil", mantendo o comportamento autístico como manifestação da esquizofrenia infantil; no DSM III o diagnóstico de "Esquizofrenia tipo infantil" desapareceu e foi realocado em "Distúrbios que habitualmente se manifestam na primeira infância ou adolescência". Assim, o autismo saiu da condição de sintoma para ser nomeado Distúrbio Autista.

No DSM IV, o autismo deixou de ser nomeado como distúrbio e passou à condição de transtorno e se manteve como entidade nosográfica dos Transtornos Globais do Desenvolvimento (TGD). Nesse eixo, encontravam-se o Transtorno Autista, Transtorno de Asperger, Transtorno de Rett, Transtorno Desintegrativo da Infância e TGD sem outra especificação. $\mathrm{Na}$ última edição do manual DSM V, o TEA englobou transtornos antes chamados de autismo infantil precoce, autismo infantil, autismo de Kanner, autismo de alto funcionamento, autismo atípico, transtorno global do desenvolvimento sem outra especificação, transtorno desintegrativo da infância e transtorno Asperger (Mas, 2018).

Essas mudanças foram implementadas buscando melhorar a sensibilidade e a especificidade dos critérios para o diagnóstico de TEA, sendo que essas modificações foram pautadas em evidências clínicas e empíricas que apontavam para a dimensionalidade de suas características cognitivas e psicológicas. A palavra espectro tem por objetivo explicitar a ideia de um contínuo associado aos transtornos neuropsiquiátricos (APA, 2014; Júlio-Costa \& Antunes, 2017). Os sintomas se encontram em um continuum único de prejuízos, com intensidades que vão de leve a grave nos domínios de comunicação social e dos comportamentos restritivos e repetitivos, em vez de constituir transtornos distintos.

Trata-se de um transtorno do neurodesenvolvimento que costuma ser reconhecido durante o segundo ano de vida, 12 a 24 meses, cujos sinais podem incluir atrasos precoces no desenvolvimento ou perda de habilidades adquiridas anteriormente (APA, 2014). Sua origem é multifatorial, sendo necessário examinar e compreender aspectos do perfil genético familiar, social e emocional no qual o indivíduo está inserido. A descrição do indivíduo com TEA é de suma importância para o delineamento do prognóstico, bem como planejamento de intervenções e estimulações, devendo ser considerados fatores como atrasos na linguagem, deficiência intelectual, déficits na coordenação motora fina e sensorial (Bosa \& Teixeira, 2017).

O diagnóstico do TEA é fundamentalmente clínico, baseado em observações das características comportamentais e a partir de informações dos pais e/ou cuidadores. Nesse sentido, os instrumentos de triagem, escalas e avaliações padronizadas vem se mostrando necessários no processo diagnóstico (Machado, Lerner, Novaes, Palladino, \& Cunha, 2014).

A literatura científica internacional apresenta instrumentos sistematizados de avaliação, como Autism Diagnostic
Interview-Revised (ADI-R) e Autism Diagnostic Observation Schedule-Generic (ADOS), ambos considerados como padrão-ouro para o diagnóstico do TEA, já com estudos preliminares de validação no Brasil (Marques \& Bosa, 2015). O Protocolo de Avaliação para Crianças com Suspeita de Transtornos do Espectro do Autismo (PROTEA-R) é um instrumento não verbal de avaliação para crianças entre 24 e 60 meses de idade, construído por pesquisadores brasileiros (Bosa, Zanon, \& Backes, 2016).

No que tange aos instrumentos de triagem do TEA no Brasil, tem-se alguns traduzidos, adaptados e validados, como a Autistic Traits of Evaluation Scale (ATA); Autism Behavior Checklist (ABC); Childhood Autism Rating Scale (CARS); Autism Screening Questionnaire (ASQ); Modified Checklist for Autism in Toddlers (M-CHAT) (Marques \& Bosa, 2015). Questões importantes sobre instrumentos de avaliação psicológica no contexto brasileiro devem ser consideradas, como os processos de tradução e validação, uso restrito a especialistas e direitos autorais (Machado et al., 2014).

Frente às dificuldades e restrições que permeiam traduções, adaptações e validações de instrumentos (Pasquali, 2010; Hutz, Bandeira, \& Trentini, 2015), por vezes, opta-se pela construção de novas técnicas de avaliação psicológica dentro de nossa realidade. Contudo, tanto no processo de construção, como no de adaptação dos instrumentos psicológicos, deve-se considerar a Resolução n ${ }^{\circ}$ 009/2018 do Conselho Federal de Psicologia (CFP), as orientações da International Test Commission (ITC), da American Education Rearch Association (AERA), da American Psychological Association (APA) e do National Concil on Measurement in Education (NCME).

Com o intuito de garantir o compromisso ético dos psicólogos na utilização de instrumentos no âmbito profissional, o CFP criou, em 2003 o Sistema de Avaliação de Testes Psicológicos (SATEPSI), que estabelece diretrizes sobre a Avaliação Psicológica e avalia a qualidade técnica-científica de instrumentos psicológicos. Esse sistema é alimentado pela Comissão Consultiva em Avaliação Psicológica do CFP que, além de avaliar a qualidade técnico-científica dos testes psicológicos, também é responsável pelas normativas que regulamentam a avaliação psicológica no contexto do profissional psicólogo.

Os instrumentos de avaliação psicológica com parecer favorável do SATEPSI, encontram-se listados no site http://satepsi.cfp.org.br/, nos campos "Testes Psicológicos Favoráveis" e "Instrumentos Não Privativos do Psicólogo". De acordo com a Resolução no 09/2018, é considerada falta ética do profissional psicólogo a utilização de testes psicológicos com parecer desfavorável ou não avaliados pelo SATEPSI, salvo para os casos de pesquisa e de ensino com objetivo formativo e histórico na Psicologia.

A submissão de instrumentos junto ao SATEPSI está condicionada a condições mínimas obrigatórias, como descrição geral do instrumento (definição e identificação dos construtos) e requisitos técnicos (procedimento de 
adaptação, quando traduzido de outra língua; fundamentação teórica; análise dos itens; precisão; validade; e sistema de correção e interpretação dos escores obtidos no estudo brasileiro). Outro critério a ser considerado nos instrumentos é o tamanho da amostra utilizada em seu estudo frente ao tamanho da população (Cozby, 2003).

Diante da relevância dos TEA, na prática clínica cotidiana e da necessidade de aprofundamento investigativo sobre o tema na realidade do Brasil, procurou-se realizar revisão sistemática da literatura científica a respeito dos instrumentos de avaliação psicológica desse tipo de transtorno, trazendo à tona similaridades, divergências e lacunas que podem contribuir para futuras investigações. O presente estudo objetivou revisar sistematicamente a literatura científica acerca dos instrumentos validados no Brasil para a caracterização do TEA, segundo relato de responsáveis e/ou outros educadores.

\section{Método}

\section{Delineamento}

Trata-se de uma revisão da literatura científica baseada no questionamento da existência de instrumentos validados, para o contexto brasileiro, referentes ao Transtorno do Espectro Autista (TEA), segundo relato de responsáveis e/ou cuidadores. Tem-se que revisões sistemáticas devem ser baseadas em perguntas objetivas, utilizando métodos sistematizados e explícitos que possam identificar, selecionar e avaliar criticamente pesquisas relevantes do assunto foco da pesquisa. Para tanto, no presente estudo, foi utilizado o método PRISMA (Principais Itens para Relatar Revisões sistemáticas e Meta-análises), a saber, um checklist com 27 itens e um fluxograma com quatro itens, que auxiliam na execução e qualidade de revisões sistemáticas e meta-análises (Galvão, Pansani, \& Harrad, 2015).

\section{Materiais}

O objeto de análise neste trabalho foram artigos de revistas científicas que visaram traduzir, adaptar e validar instrumentos para a caracterização do TEA, encontrados em bases de dados, abrangendo os últimos dez anos. Aplicaram-se restrições à cronologia e à língua original de publicação.

\section{Procedimentos}

Para realizar esta revisão sistemática, foram utilizadas as diretrizes do PRISMA (Principais Itens para Relatar Revisões sistemáticas e meta-análises), como o checklist de itens a serem incluídos e o fluxograma com as diferentes fases de uma revisão sistemática da literatura científica. Foram consultadas as bases de dados: Embase, PubMed, Scopus e BVS, da data de publicação até março de 2019. Nas três primeiras bases, os documentos potencialmente relevantes foram selecionados com os seguintes descritores: "autism", "autistic", "instrument", "scale", "questionnaire", "child", "child's", "children", "children's", "teacher" e descritores não aceitos: "adolescent", "adolescence's" e "adolescence". Na base de dados da BVS se aplicou os descritores: "autism", "autista", "instrument", "escala", "scale", "questionário", "questionnaire", "child" e "children". Foram usados os seguintes operadores booleanos: and entre os descritores, or para as variações de um mesmo descritor e not para descritores excluídos na combinação.

Foram aceitos artigos publicados entre 2009 a março de 2019, textos completos, revisados por pares e nos seguintes idiomas: inglês, espanhol e português. A seleção dos estudos foi baseada no título e resumo, e a extração dos dados na análise dos artigos completos. Critérios de inclusão: 1. artigos que investigavam propriedades psicométricas do instrumento no contexto do Brasil (validação, tradução, precisão ou adaptação); 2. artigos que tratavam de instrumentos para caracterização do TEA; 3. artigos onde a população-alvo estava entre seis e 12 anos de idade; e 4. artigos onde ao menos um dos informantes ou respondentes não fosse especialista (responsáveis e/ou educadores). Critérios de exclusão: 1. artigos repetidos; 2. artigos não disponíveis na íntegra; 3. artigos em línguas diferentes do português, inglês e espanhol; e 4. artigos que utilizavam amostras de outros países.

Para amenizar o risco de viés, dois juízes realizaram os procedimentos de seleção e extração de artigos, de forma independente, com índice de concordância de 94\%. Em caso de ausência de acordo quanto aos estudos selecionados e informações extraídas, um especialista foi consultado para buscar decisão sobre as variáveis em foco. Com base na análise final da seleção, os estudos foram caracterizados de acordo com seus autores e ano de publicação, instrumentos utilizados, objetivo, participantes e quais respondentes e/ou informantes foram utilizados para responder aos instrumentos. A Figura 1 representa o fluxograma detalhado do processo de seleção de artigos científicos utilizados neste estudo.

A Figura 1 sinaliza que a busca inicial nas bases de dados identificou 705 arquivos, que foram lançados na página do EndNote para a organização das referências bibliográficas. O programa encontrou 204 artigos duplicados, sendo assim, 501 artigos estavam disponíveis para a leitura do título e resumo. Nessa triagem, os textos selecionados atendiam os seguintes critérios: textos completos; investigação das propriedades psicométricas dos instrumentos (validação, tradução, precisão ou adaptação); instrumentos tinham por objetivo a caracterização do TEA; a população-alvo compreendia a idade entre seis e 12 anos; e, ao menos, um dos informantes ou respondentes, carecia de ser um não especialista (responsáveis e/ou educadores). Após essa primeira seleção, foram excluídos 446 artigos, consequentemente 55 artigos foram direcionados para a leitura na íntegra e verificação dos critérios de inclusão e exclusão descritos nos procedimentos, chegando-se a um número final de oito trabalhos. 


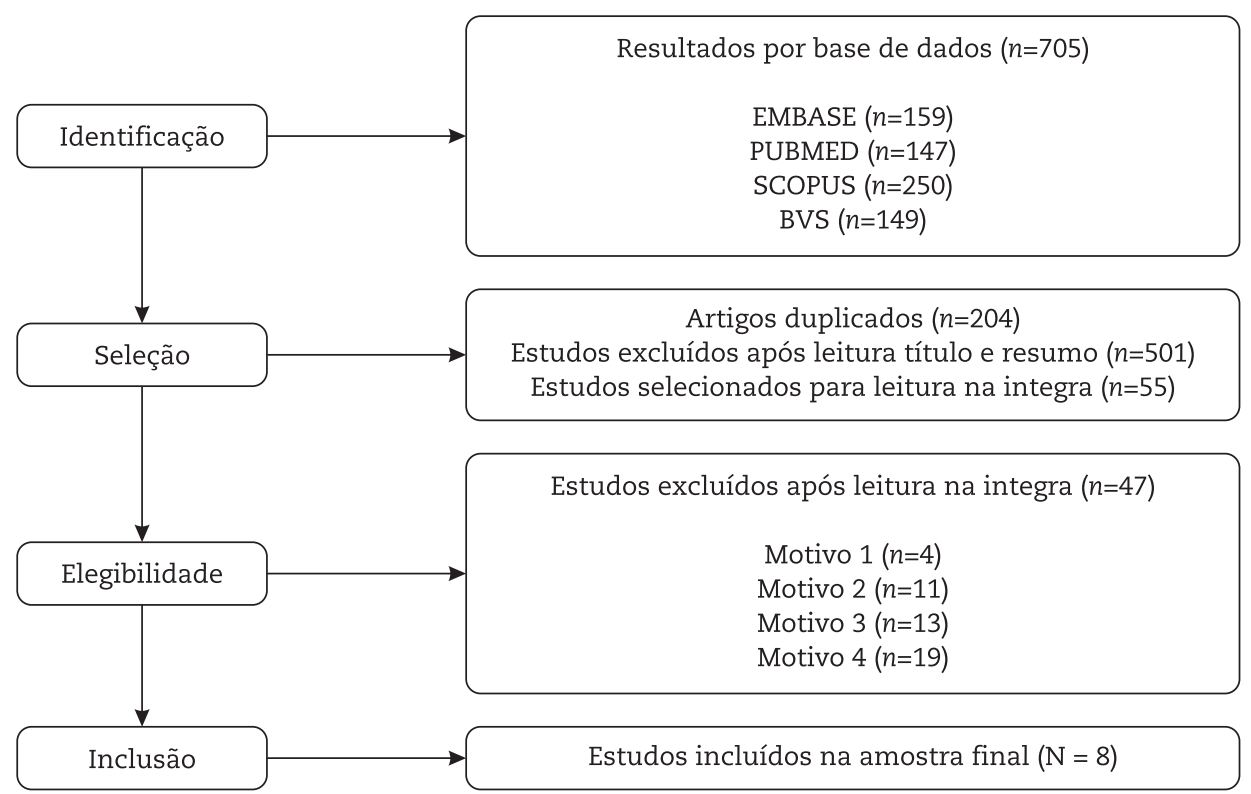

Figura 1. Fluxograma dos estudos selecionados para a revisão de literatura científica

Nota. Motivo 1 - Amostra não contemplava a faixa etária de 6 e 11 anos; Motivo 2 - Instrumentos respondidos por profissionais ou especialistas; Motivo 3 - Instrumento não investigou propriedades psicométricas para a caracterização do TEA; Motivo 4 - Estudo com amostra de outro país que não o Brasil

\section{Resultados}

Inicialmente encontram-se descritos os oito artigos selecionados segundo o título dos estudos, respectivos autores e data de publicação. Essas informações constam na Tabela 1.

Em uma busca realizada no site do SATEPSI em março de 2019, utilizando as listas "Testes Psicológicos Favoráveis" e "Instrumentos Não Privativos do Psicólogo", os instrumentos listados na Tabela 1 foram lançados na janela "Nome" seguindo os nomes completos dos instrumentos na língua de origem e suas abreviações. Cabe destacar que nenhum dos instrumentos identificados na literatura científica foi encontrado no referido site.

A seguir constam informações sobre objetivos dos estudos, instrumentos utilizados no processo de validação, tradução e/ou adaptação, tamanho e faixa etária da amostra, e quais informantes responderam ao instrumento. Esses dados compõem a Tabela 2.

Tabela 1

Títulos dos Estudos sobre Instrumentos de Avaliação do TEA Segundo Pais e/ou Cuidadores entre 2009-2019

\begin{tabular}{lc}
\multicolumn{1}{c}{ Título do artigo científico } & Autores \\
\hline $\begin{array}{l}\text { Questionário sobre dificuldades comunicativas percebidas } \\
\text { por pais de crianças do espectro do autismo }\end{array}$ & Balestro e Fernandes (2012) \\
$\begin{array}{l}\text { Translation, adaptation, and preliminary validation of the Brazilian } \\
\text { version of the Behavior Problems Inventory (BPI-01) }\end{array}$ & Baraldi, Rojahn, Seabra, \\
Carreiro e Teixeira (2013)
\end{tabular}


Tabela 1 (continuação)

Títulos dos Estudos sobre Instrumentos de Avaliação do TEA Segundo Pais e/ou Cuidadores entre 2009-2019

\begin{tabular}{lc}
\hline \multicolumn{1}{c}{ Título do artigo científico } & Autores \\
\hline $\begin{array}{lc}\text { Instrument to screen cases of pervasive developmental } \\
\text { disorder - a preliminary indication of validity }\end{array}$ & Sato et al. (2009) \\
& Sousa-Morato e \\
Adaptação sociocomunicativa no espectro autístico: & Fernandes (2009) \\
\hline
\end{tabular}

Tabela 2

Objetivos, Instrumentos, Procedimentos e Amostra dos Artigos Científicos

\begin{tabular}{lc}
\hline \multicolumn{1}{c}{ Objetivos /Amostra/ Informantes } & Autores \\
\hline Elaborar um questionário para o levantamento de dificuldades comunicativas percebidas & Balestro \& \\
por pais e/ou cuidadores de crianças do espectro do autismo em relação a seus filhos. & Fernandes (2012) \\
Número de participantes $=40$ &
\end{tabular}

Pais e/ou cuidadores de crianças de 2 a 10 anos.

Descrever o processo de adaptação cultural para português do Brasil do Behavior Problems Inventory (BPI-01), bem como indicadores preliminares de sua fidedignidade e validade.

Baraldi et al. (2013)

Número de participantes $=60$

Pais e/ou cuidadores de crianças de 6 a 18 anos.

O objetivo desse trabalho foi avaliar as propriedades psicométricas da versão em português da Escala de Responsividade Social-2 (ERS-2) para crianças e adolescentes com transtorno do espectro autista (TEA).

Número de participantes $=115$

Pais de crianças e adolescentes de 4 a 18 anos.

Traduzir para o português do Brasil a ADI-R (Autism Diagnostic Interview-Revised), uma ferramenta diagnóstica extremamente útil em casos de autismo.

Becker et al.

Número de participantes $=40$

Pais e/ou cuidadores de crianças e adolescentes de 8 a 16 anos.

Traduzir o Children's Communication Checklist-2 (CCC-2) para o idioma português brasileiro, realizar sua adaptação transcultural e avaliar sua consistência interna.

Barbosa et al.

Número de participantes $=20$

Pais e/ou cuidadores de crianças e adolescentes de 4 a 16 anos.

Adaptar o instrumento Indicadores Clínicos de Risco para o Desenvolvimento Infantil (IRDI) para questionário retrospectivo para pais de crianças de 3 a 7 anos e aplicá-lo em dois grupos de sujeitos.

Número de participantes $=72$

Pais de crianças de 2 a 7 anos.

Tradução, retroversão, adaptação cultural e validação do Autism Screening Questionnaire para a língua portuguesa e para o seu uso no Brasil.

Sato et al.

Número de participantes $=120$

Pais e/ou responsáveis de crianças.

Verificar a efetividade da aplicação do protocolo de adaptação sociocomunicativa a diferentes fontes de informação, nesse caso, pais e terapeutas, na identificação de diferenças individuais em crianças com Distúrbios do Espectro Autístico.

Número de participantes $=48$

Pais e terapeutas de crianças de 3 a 11 anos.

Pode-se observar, na Tabela 2, que todos os estudos encontrados tinham por objetivo verificar propriedades psicométricas dos instrumentos e/ou validá-los em amostra brasileira. Os instrumentos variaram entre escalas, 
questionários e protocolos, utilizados na avaliação ou triagem de rastreio dos sintomas do TEA. Os estudos apresentaram similaridades, como processos de validação de instrumentos, amostra-alvo com pais e/ou cuidadores de crianças e apresentação de dados estatísticos extraídos da aplicação do instrumento. É importante apontar que houve grande variabilidade entre amostras no que tange a faixa etária, variando entre dois e 18 anos, e o tamanho da amostra, entre 20 e 120 participantes. Sete estudos utilizaram como fonte os pais e/ou cuidadores das crianças e um estudo comparou os informantes pais e terapeutas.

A ERS-2 é uma escala que pode ser aplicada aos pais ou aos professores em sua versão original, contudo, o estudo de validação, apresentado por Barbosa et al. (2015), não contempla os professores como informantes na amostra. Isso evidencia que nenhum estudo apresentado utilizou os educadores como fonte para a coleta de informações dos instrumentos.

O procedimento de coleta de dados nos estudos envolveu ambientes diversos, desde ambulatórios de hospitais públicos a consultórios privados. O estudo com a escala ERS-2 (Barbosa et al., 2015) incluiu o diagnóstico de TEA segundo o DSM-V, enquanto os demais trabalhos contaram com os critérios diagnósticos do manual DSM-IV. No que se refere ao diagnóstico, cinco estudos relataram que foram realizados por profissionais neurologistas, pediatras, geneticistas ou psiquiatras (Baraldi et al., 2013; Barbosa et al., 2015; Becker et al., 2012; Sato et al., 2009; Sousa-Morato \& Fernandes, 2009); dois estudos informaram que os pacientes frequentaram o ambulatório de psiquiatria, mas não especificaram o profissional responsável pelo diagnóstico (Costa et al., 2013; Machado et al., 2014); e um estudo não informou os profissionais responsáveis pelo diagnóstico (Balestro \& Fernandes, 2012).

No que tange ao delineamento de análises comparativas entre grupos, quatro estudos compararam crianças com autismo e crianças típicas sem queixas ou histórico de transtornos (Balestro \& Fernandes, 2012; Baraldi et al., 2013; Barbosa et al., 2015; Machado et al., 2014); um estudo comparou grupo com TEA e grupo com Deficiência Intelectual moderada (Becker et al., 2012); um estudo dividiu os grupos em transtorno invasivos do desenvolvimento, síndrome de Down e um grupo com outros transtornos psiquiátricos (Sato et al., 2009). Por fim, dois estudos não realizaram comparações entre grupos clínico e controle (Costa et al., 2013; Sousa-Morato \& Fernandes, 2009).

Com relação aos construtos e variáveis investigadas nos estudos, tem-se a sistematização das informações listada na Tabela 3.

Tabela 3

Construtos e Variáveis Investigadas nos Estudos Identificados

\begin{tabular}{lc}
\hline \multicolumn{1}{c}{ Variáveis Avaliadas } & Autores \\
\hline Dificuldades de linguagem. & Balestro e Fernandes (2012) \\
Comportamento autolesivo, estereotipado e comportamento agressivo. & Baraldi et al. (2013) \\
$\begin{array}{l}\text { Prejuízos de socialização, comunicação, } \\
\text { comportamentos repetitivos e interesses restritos. }\end{array}$ & Barbosa et al. (2015) \\
$\begin{array}{l}\text { Interação social; comunicação; padrões de comportamento } \\
\text { repetitivos e estereotipados; interesses restritos. }\end{array}$ & Becker et al. (2012) \\
$\begin{array}{l}\text { Aspectos da comunicação como fala, vocabulário, estrutura da frase e habilidades } \\
\text { de linguagem social de crianças e adolescentes que falam por meio de sentenças. }\end{array}$ & Costa al. (2013) \\
Não especificado; problemas no desenvolvimento infantil. & Machado et al. (2014) \\
$\begin{array}{l}\text { Prejuízos de socialização, comunicação, } \\
\text { comportamentos repetitivos e interesses restritos. }\end{array}$ & Sato et al. (2009) \\
Linguagem e socialização. & Sousa-Morato e \\
\end{tabular}

A Tabela 3 sinaliza que os critérios diagnósticos estabelecidos no TEA variaram entre os instrumentos apresentados. Dois instrumentos avaliaram as dificuldades em linguagem (Balestro \& Fernandes, 2012; Costa et al., 2013); três estudos avaliaram os prejuízos de socialização, comunicação, comportamentos repetitivos e estereotipados, e interesses restritos (Barbosa et al., 2015; Becker et al. 2012; Sato et al., 2009); um estudo avaliou comportamentos autolesivos, estereotipados e comportamento agressivo (Baraldi et al., 2013); um estudo avaliou a 
linguagem e socialização (Sousa-Morato \& Fernandes, 2009) e um estudo não especificou as variáveis avaliadas, descrevendo-as como problemas no desenvolvimento infantil (Machado et al., 2014).
Por fim considerou-se adequado realizar uma síntese dos principais resultados referentes às evidências estatísticas apresentadas nos estudos. Esses dados compõem a Tabela 4 .

Tabela 4

Principais Resultados Empíricos e Evidências Estatísticas dos Estudos

\begin{tabular}{lc}
\hline Evidências / Principais achados & Autores \\
\hline A análise inferencial evidenciou diferença em 19 das 24 questões, exceto nas questões 7, 10, & Balestro \& \\
12, 13 e 24. Nessa análise preliminar, as respostas à questão 25 (aberta) não foram analisadas. & Fernandes (2012)
\end{tabular}

Utilizou-se o teste $t$ de Student, o valor de p variou de $<0,001$ a 0,2. Por não se tratar de um

teste e sim de um questionário, as variáveis psicométricas não se aplicam como critério de

julgamento de confiabilidade. A análise do questionário não objetiva um número que diferencie

um sujeito do outro, mas conhecer as dificuldades percebidas pelos pais e/ou cuidadores.

A consistência interna do BPI-01 foi satisfatória, com alfa de Cronbach de 0,65 para a escala de comportamentos autoagressivos, 0,82 para comportamentos estereotipados e 0,91 para comportamentos agressivos/destrutivos. Considerando-se uma frequência média de 0,5, a curva Receiver Operating Characteristic (ROC) verificou indicadores do instrumento com $80 \%$ de sensibilidade e $3 \%$ de especificidade na escala de comportamento estereotipado, $50 \%$ de sensibilidade e $10 \%$ de especificidade na escala de comportamento agressivo/destrutivo e $76 \%$ de sensibilidade e $6 \%$ de especificidade na escala de comportamento autolesivo. Foram verificadas correlações de baixas a moderadas entre os escores do BPI-01, do ASQ e do CBCL/6-18.

A ERS-2 demonstrou boa consistência interna (alfa de Cronbach =0,952), um ponto de corte de 41 , sensibilidade de $96,8 \%$, especificidade de $100,0 \%$, valor preditivo positivo de $100,0 \%$ e valor preditivo negativo de 99,9\% para a identificação de TEA. Alguns itens se apresentaram pouco consistentes do ponto de vista estatístico (correlação item-total negativas e misfitting). O mapa de itens mostrou má cobertura da variável latente, especialmente no espectro mais leve do TEA.

A consistência interna foi alta, com um $\alpha$ de Cronbach de 0,976. A validade de critério mostrou uma sensibilidade e uma especificidade de 100,0\%, a partir dos critérios diagnósticos do DSM-IV. A entrevista teve alta validade discriminante, com maiores escores no grupo de pacientes com autismo, bem como alta consistência entre observadores, com Kappa médio de 0,824.

A confiabilidade do instrumento é aceitável, os valores do alfa de Cronbach variaram de 0,75 a 0,90. O valor de alfa de Cronbach para o CCC-2 foi de 0,93.

Baraldi

et al. (2013)

Barbosa et al.

Becker

et al. (2012)

Costa et al.

(2013)

Machado et al. (2014)

31 questões do instrumento, obteve-se valor de 0,89 por alfa de Cronbach. A comparação entre os grupos, segundo as respostas dos pais às 31 questões do IRDI-questionário, mostrou diferença estatisticamente significativa em 16 delas.

Valores de sensibilidade de $92,5 \%$ e especificidade de $95,5 \%$ foram encontrados para uma pontuação de 15 , como sendo um valor discriminativo para os sujeitos com características de transtornos globais do desenvolvimento/transtornos invasivos do desenvolvimento. A validade interna para o total das 40 questões foi de 0,895, com uma variação entre 0,6 a 0,8. Os valores de confiabilidade obtidos pelo teste e reteste demonstraram que a maioria das questões obteve alta concordância.

Genericamente, pode-se dizer que as respostas sobre a adaptação sociocomunicativa, obtidas por meio de diferentes fontes de informação, ou seja, pais e terapeutas foram semelhantes. Analisando os dados brutos, observa-se que os pais apresentaram consistentemente um número maior de respostas positivas do que as terapeutas para as questões relativas ao desempenho social.

Na Tabela 4, observa-se variabilidade na apresentação dos dados estatísticos: dois estudos apresentaram resultados baseadas em análises utilizando o teste $\mathrm{t}$ de Student (Balestro \& Fernandes, 2012; Sousa-Morato \& Fernandes 2009); seis estudos esboçaram a consistência interna dos instrumentos por meio do cálculo de alfa de Cronbach (Baraldi et al., 2013; Barbosa et al., 2015; Becker et al., 2012; Costa et al., 2013; Machado et al., 2014; Sato et al., 2009). Quatro estudos calcularam sensibilidade e especificidade (Baraldi et al., 2013; Barbosa et 
al., 2015; Becker et al., 2012; Sato et al., 2009); um estudo apresentou o cálculo de consistência entre observadores (Becker et al., 2012); um estudo apresentou o ponto de corte e o valor preditivo (Barbosa et al., 2015); dois estudos verificaram as correlações obtidas com outros instrumentos (Baraldi et al., 2013; Sato et al., 2009); e um estudo realizou teste e reteste (Sato et al., 2009).

Outros resultados encontrados referem-se às limitações técnico-científicas apontadas pelos próprios autores. Balestro e Fernandes (2012) expõem que um dos obstáculos está correlacionado aos critérios de inclusão dos sujeitos no grupo sem queixas de linguagem em comparação ao grupo com autismo. Os estudos de Baraldi et al. (2013), Becker et al. (2012) e Machado et al. (2014) apontam o tamanho da amostra como uma possível limitação, amostras menores que 75 participantes, e isso pode superestimar as propriedades psicométricas dos instrumentos. $\mathrm{O}$ estudo de Baraldi et al. (2013) discutiu a necessidade de se calcular correlações para a frequência e severidade de cada problema de comportamento. Machado et al. (2014) ressaltam que o instrumento IRDI não é específico para a avaliação do autismo, mas para os problemas no desenvolvimento infantil amplamente. Barbosa et al. (2015) observam que novos estudos são necessários com grupos que discriminem os especificadores de nível de desenvolvimento intelectual e de linguagem, pois alguns itens da escala podem não avaliar o TEA, mas outros problemas associados. Costa et al. (2013) citam como entraves as variações em subescalas e baixa consistência interna em estudos anteriores; e, para que o teste possa ser amplamente utilizado em ambientes clínicos e de pesquisa, ainda há necessidade de validação da versão brasileira do instrumento. No estudo de Sato et al. (2009), os autores apresentam que o instrumento detém direitos autorais e seu uso não é isento de custos; poucos estudos na literatura utilizaram esse instrumento, o que dificulta a comparação dos resultados. Sousa-Morato e Fernandes (2009) mencionam como limitações a não caracterização dos aspectos: nível de escolaridade dos respondentes, nível intelectual das crianças e tempo de exposição.

\section{Discussão}

Com o objetivo de revisar sistematicamente a literatura científica acerca dos instrumentos validados no Brasil para a caracterização do TEA, segundo relato de responsáveis e/ou outros educadores, o presente estudo traz contribuições no que tange ao panorama atual da área. Aponta limitações encontradas nos trabalhos que podem auxiliar próximos estudos no sentido de desenvolver instrumentos que possam ser submetidos às normativas do CFP e, posteriormente, apresentados no SATEPSI.

Um ponto inicial, a ser considerado na elaboração e/ou validação de instrumentos, é a indicação do grupo-critério (baseadas nas classificações diagnósticas) que irá estabelecer quais comportamentos ou itens descrevem os participantes e seu pertencimento a um grupo. As classificações psiquiátricas das doenças mentais variam de acordo com as construções dos manuais; nesse sentido, se as definições de doenças mentais são diferentes, os testes construídos para avaliá-las serão diferentes, pois os grupos-critérios são diferentemente definidos e classificados (Pasquali, 2010). Dos instrumentos demonstrados, apenas a ERS-2 é construída de acordo com os critérios diagnósticos do DSM-V, os demais são estruturados nas versões anteriores do manual. Torna-se clara a importância de revisão dos estudos, segundo os critérios diagnósticos vigentes. Da mesma forma, esse dado reforça o apontado nas normativas do CFP sobre avaliação psicológica e, em especial, a Resolução 09/2018, que estabelece a necessidade de revisão dos estudos com evidências de validade e normas a cada 15 anos. Esse aspecto também serve como forma de garantir a atualização dos testes psicológicos em termos técnico-científicos.

Outro ponto a ser destacado é que os instrumentos, em sua maioria, advêm de estudos de outros países. Hutz, Bandeira e Trentini (2015) ressaltam que esse processo oferece como vantagem a possibilidade de comparações transculturais, em compensação alguns itens podem não refletir o traço latente tanto quanto o instrumento original e as adaptações podem não ser suficientes. Os dados apontaram que quatro estudos descrevem em seus objetivos a tradução para o idioma português brasileiro e a adaptação transcultural (Barbosa et al., 2015; Becker et al. 2012; Costa et al., 2013; Sato et al., 2009).

No que tange a idade alvo dos instrumentos, mesmo tendo restringido o intervalo foco de 6-12 anos como critério de inclusão nessa revisão, nota-se uma variação na idade das amostras estudadas. Verifica-se que a menor idade encontrada é de dois anos e a maior de 18 anos, assim, um instrumento propõem-se a avaliar um mesmo construto em diferentes etapas, desse modo, o traço pode se alterar entre as fases do desenvolvimento.

Observa-se nos estudos encontrados nesta revisão amostras de 20 a 120 pessoas (considerando a divisão em grupo clínico e controle), sendo a maior amostra de 90 participantes (crianças ou adolescentes). Esses indicadores são menores do que os indicados pela literatura científica. Cozby (2003) afirma que, dentro de um intervalo de confiança de $95 \%$, a representação de uma população com mais de 100.000 indivíduos é equivalente a uma amostra mínima de 96 pessoas, amostras maiores e mais representativas variam de 384 a 1.067 pessoas.

Estudos isolados não esgotam as possibilidades de validação de instrumentos, portanto, novas investigações sobre um mesmo instrumento podem fornecer dados estatísticos suficientes para a adequada análise do TEA. Ressalta-se que, para a utilização desses instrumentos no ambiente profissional, devem-se considerar os direitos autorais, a comercialização ou a disponibilização pelos autores e as normas vigentes do CFP. Adverte-se que o uso de instrumentos implica a 
existência de direitos autorais, e os estudos de validação podem não ser liberados para uso no ambiente clínico ou projetos de investigação, caso o autor não disponibilize a autorização (Backes, Mônego, Bosa \& Bandeira, 2014). Dessa forma, a carência de informações explícitas sobre direitos autorais envolve riscos em relação à utilização desses instrumentos.

A busca realizada no site do CFP apontou que os instrumentos mencionados nesta revisão da literatura científica ainda não foram submetidos ao SATEPSI para uso profissional do psicólogo, tampouco são comercializados, estando disponíveis apenas em trabalhos científicos, como artigos, dissertações e teses. Isso sugere que o profissional psicólogo está habilitado a manuseá-los em pesquisas, considerando a autorização formal do editor, contudo, o uso dos instrumentos na prática profissional é considerado antiético por não considerar as premissas da Resolução no 009/2018 do CFP.

Em suma, o presente estudo aponta para importantes questões éticas e técnicas para o campo do TEA. A análise apresentada neste estudo teve como objetivo fornecer informações aos profissionais psicólogos sobre os instrumentos utilizados na avaliação do TEA no Brasil nos últimos dez anos e suas implicações no uso profissional e de pesquisa. Estudos futuros deveriam centrar atenção para o desenvolvimento de escalas para avaliação de TEA em população brasileira para uso profissional do psicólogo.

\section{Referências}

American Psychological Association [APA]. (2014). Manual diagnóstico de transtornos mentais: DSM-V (5a ed.). Porto Alegre: Artmed.

Backes, B., Mônego, B. G., Bosa, C. A., \& Bandeira, D. R. (2014). Psychometric properties of assessment instruments for autism spectrum disorder: a systematic review of Brazilian studies. Jornal Brasileiro de Psiquiatria, 63(2),154-64. doi:10.1590/0047-2085000000020

^Balestro, J. I., \& Fernandes, F. D. M. (2012). Questionário sobre dificuldades comunicativas percebidas por pais de crianças do espectro do autismo. Revista da Sociedade Brasileira de Fonoaudiologia, 17(3), 279-286. doi: 10.1590/s1516-80342012000300008

^Baraldi, G. da S., Rojahn, J., Seabra, A. G., Carreiro, L. R. R., \& Teixeira, M. C. T. V. (2013). Translation, adaptation, and preliminary validation of the Brazilian version of the Behavior Problems Inventory (BPI-01). Trends in Psychiatry and Psychotherapy, 35(3), 198-211. doi: 10.1590/s2237-60892013000300007

^Barbosa, I. G., Rodrigues, D. H., Rocha, N. P., Simões-e-Silva, A. C., Teixeira, A. L., \& Kummer, A. (2015). Propriedades psicométricas da Escala de Responsividade Social-2 para Transtornos do Espectro Autista. Jornal Brasileiro de Psiquiatria, 64(3), 230-237. doi: 10.1590/00472085000000083

^Becker, M. M., Wagner, M. B., Bosa, C. A., Schmidt, C., Longo, D., Papaleo, C., \& Riesgo, R. S. (2012). Tradução e validação da ADI-R (Autism Diagnostic Interview-Revised) para diagnóstico de autismo no Brasil. Arquivos de Neuro-Psiquiatria, 70(3), 185-190. doi: 10.1590/S0004-282X2012000300006

Bosa, C. A., \& Teixeira, M. C. T. V. (2017). Autismo: Avaliação psicológica e neuropsicológica (1 ed). São Paulo: Hogrefe.

Bosa, C. A., Zanon, R. B., \& Backes, B. (2016). Autismo: Construção de um protocolo de avaliação do comportamento da criança - Protea R. Psicologia - Teoria e Prática, 18(1), 194-205. doi: 10.15348/1980-6906/psicologia.v18n1p194-205

*Costa, V. B. S. da, Harsányi, E., Martins-Reis, V. de O., \& Kummer, A. (2013). Tradução e adaptação transcultural para o português brasileiro do teste Children's Communication Checklist-2. CoDAS, 25(2), 115-119. doi: 10.1590/s2317-17822013000200005

Cozby, C. P. (2003). Métodos de pesquisa em ciência do comportamento. São Paulo: Atlas.

Galvão, T. F., Pansani; T. de S. A., \& Harrad, D. (2015). Principais itens para relatar Revisões sistemáticas e Meta-análises: A recomendação PRISMA. Epidemiologia e Serviços de Saúde, 24(2), 335-342. doi: 10.5123/s1679-49742015000200017

Hutz, C. S., Bandeira, D. R., \& Trentini, C. M. (2015). Psicometria. Porto Alegre: Artmed.

Júlio-Costa, A., \& Antunes, A. M. (2017). Transtorno do espectro autista na prática clínica. São Paulo: Pearson Clinical Brasil.

Machado, F., Lerner, R., Novaes, B. C. A. C., Palladino, R. R. R., \& Cunha, M. C. (2014). Questionário de indicadores clínicos de risco para o desenvolvimento infantil: avaliação da sensibilidade para transtornos do espectro do autismo. Audiology - Communication Research, 19(4):345-51. doi: 10.1590/S2317-64312014000300001392

*Machado, F. P., Palladino, R. R. R., \& Cunha, M. C. (2014). Adaptation of the Child Development Clinical Risk Indicators instrument to retrospective parent report. Codas, 26(2), 138-147. doi: 10.1590/2317-1782/2014001IN

Marques, D. F., \& Bosa, C. A. (2015). Protocolo de avaliação de crianças com autismo: Evidências de validade de critério. Psicologia: Teoria e Pesquisa, 31(1), 43-51. doi: 10.1590/0102-37722015011085043051

Mas, N. A. (2018). Transtorno do Espectro Autista - história da construção de um diagnóstico. (Dissertão de Mestrado), Universidade de São Paulo, São Paulo - SP, Brasil. Recuperado de https://teses.usp.br/teses/disponiveis/47/47133/tde-26102018-191739/publico/mas_me.pdf

Pasquali, L. (2010). Instrumentação psicológica: Fundamentos e práticas. Porto Alegre: Artmed.

^Sato, F. P., Paula, C. S., Lowenthal, R., Nakano, E. Y., Brunoni, D., Schwartzman, J. S., \& Mercadante, M. T. (2009). Instrument to screen cases of pervasive developmental disorder: a preliminary indication of validity. Revista Brasileira de Psiquiatria, 31(1), 30-33. doi: 10.1590/ s1516-44462009000100008

*Sousa-Morato, P. F., \& Fernandes, F. D. M. (2009). Adaptação sócio-comunicativa no espectro autístico: Dados obtidos com pais e terapeutas. Revista da Sociedade Brasileira de Fonoaudiologia, 14(2), 225-233. doi:10.1590/s1516-80342009000200014

\section{Sobre as autoras}

Camila Costa e Silva é psicóloga, graduada em Psicologia pela Pontifícia Universidade Católica de Goiás (PUC-Goiás). Atualmente, é Mestranda no Programa de Pós-Graduação Stricto Sensu em Psicologia da PUC-Goiás.

Luciana Carla dos Santos Elias é doutora em Psicologia pela Faculdade de Filosofia, Ciências e Letras de Ribeirão Preto-USP. Atualmente docente junto ao Departamento de Psicologia da FFCLRP-USP e ao Programa de Pós-graduação em Psicologia da FFCLRP-USP. 